\title{
CDISC SDTM Microbiology Susceptibility Test Name Terminology
}

National Cancer Institute

\section{Source}

National Cancer Institute. CDISC SDTM Microbiology Susceptibility Test Name

Terminology. NCl Thesaurus. Code C128687.

Terminology associated with the microbiology susceptibility test name codelist of the Clinical Data Interchange Standards Consortium (CDISC) Study Data Tabulation Model (SDTM). 